\title{
Erratum to: Enhancing the Antitumor Activity of Berberine Hydrochloride by Solid Lipid Nanoparticle Encapsulation
}

Lu Wang, ${ }^{1}$ Hongtao Li, ${ }^{2}$ Shengpeng Wang, ${ }^{1}$ Rong Liu, ${ }^{2}$ Zhisheng Wu, ${ }^{3}$ Chunming Wang, ${ }^{1,4}$

Yitao Wang, ${ }^{1}$ and Meiwan Chen ${ }^{1,4}$

\section{Erratum to: AAPS PharmSciTech \\ DOI: 10.1208/s12249-014-0112-0}

The first parts $\mathbf{a}, \mathbf{b}$, and $\mathbf{c}$ in Fig. 1 and parts $\mathbf{a}$ and $\mathbf{b}$ in Fig. 3 should be disregarded due to article composition errors.

${ }^{1}$ State Key Laboratory of Quality Research in Chinese Medicine, Institute of Chinese Medical Sciences, University of Macau, Av. Padre Tomas Pereira S.J., Taipa, 999078, Macau, China.

${ }^{2}$ State Key Laboratory of Respiratory Diseases, First Affiliated Hospital of Guangzhou Medical College, 510120, Guangzhou, China.

${ }^{3}$ Beijing University of Chinese Medicine, 100102, Beijing, China.

${ }^{4}$ To whom correspondence should be addressed. (e-mail: CMWang@umac.mo; chenmeiwan81@163.com) 